Onkologe 2009 $\cdot 15: 1179$

DOI 10.1007/s00761-009-1725-x

Online publiziert: 20. November 2009

๑) Springer-Verlag 2009

\author{
J. Weitz ${ }^{1}$ P.M. Schlag ${ }^{2}$ \\ ${ }^{1}$ Klinik für Allgemein-, Viszeral- und Transplantationschirurgie, \\ Chirurgische Universitätsklinik Heidelberg \\ ${ }^{2}$ Charité Comprehensive Cancer Center, Charité - \\ Universitätsmedizin Berlin, Campus Mitte, Berlin
}

\title{
Kolonkarzinom - Es bleibt noch viel zu tun
}

Thema des Beitrags von $P$. Thermann und T. Seufferlein. Es zeigt sich insbesondere, welch großer Aufklärungsbedarf der Bevölkerung noch im Bereich der Primärprävention (Lebensführung) wie auch der sekundären Prävention (therapeutische Koloskopie) besteht. Die nächsten Beiträge widmen sich der Therapie des Kolonkarzinoms. Grundlage der Therapie ist die chirurgische Resektion mit einer radikulären Lymphonodektomie, wie im Beitrag von M. Koch und J. Weitz, Heidelberg dargestellt. Besonders wichtig erscheint die Tatsache, dass die Prognose der Patienten u. a. auch von der Qualität der Chirurgie und der Erfahrung des Chirurgen bzw. des Behandlungsteams abhängt. Die adjuvante Therapie des Kolonkarzinoms wird im Beitrag von G. Schuch, Hamburg; D. Arnold, Halle; C. Bokemeyer, Hamburg diskutiert; im Stadium III ist die adjuvante Therapie fest etabliert, im Stadium II dagegen derzeit nur bei bestimmten Risikokonstellationen indiziert. So standardisiert das Vorgehen à priori in der adjuvanten Therapie, so individuell ist das Management in der metastasierten Situation (Beitrag von G.Folprecht, Dresden; C.Bokemeyer, Hamburg; J.Weitz, Heidelberg). Gerade hier ist das interdisziplinäre Vorgehen von entscheidender Bedeutung, da chirurgische, interventionell-radiologische und medizinisch-onkologische Maßnahmen genau aufeinander abgestimmt bzw. gegeneinander abgewogen werden müssen. Der Beitrag von C. Rödel, Frankfurt, bezüglich der strahlentherapeutischen Aspekte der Therapie des Kolonkarzinoms rundet die Darstellung der therapeutischen Optionen ab.
Betrachtet man die einzelnen Beiträge, so lassen sich verschiedene Schlussfolgerungen ziehen: Nur durch ein besseres Wissen bezüglich der Pathogenese werden sich neue und individualisierte Therapieansätze entwickeln lassen. Die interdisziplinäre Therapie des Kolonkarzinoms mit höchster Qualität muss das Ziel aller Anstrengungen sein. Was nutzen alle diese Erkenntnisse aber, wenn sie nicht bei den Patienten ankommen? Dieses Problem zeigt sich nicht nur bei der primären und sekundären Prävention des Kolonkarzinoms. Es gibt also noch viel für uns alle gemeinsam zu tun.

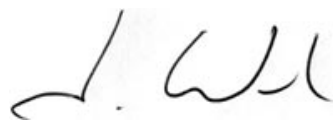

J. Weitz

Für die Herausgeber des Schwerpunktheftes

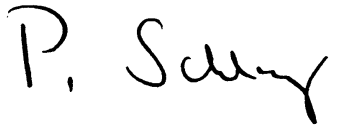

P.M. Schlag

Für die Herausgeber

\section{Korrespondenzadresse \\ Prof. Dr. J. Weitz}

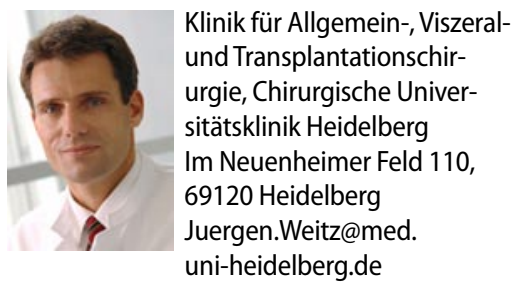

Interessenkonflikt. Der korrespondierende Autor gibt an, dass kein Interessenkonflikt besteht. keiten der primären und sekundären Prävention des Kolonkarzinoms ist spezielles 